\title{
A Time-Resolved Optical Study of the Paramagnetic Dielectric-Ferromagnetic Metal Transition in $\mathrm{La}_{0.7} \mathrm{Ca}_{0.3} \mathrm{MnO}_{3}$
}

\author{
O. V. Misochko ${ }^{\mathrm{a}, *}$, E. M. Kaidashev ${ }^{\mathrm{b}}$, N. Georgiev, \\ T. Dekorsyc ${ }^{c}$, and I. N. Zakharchenko ${ }^{d}$ \\ anstitute of Solid-State Physics, Russian Academy of Sciences, \\ Chernogolovka, Moscow oblast, 142432 Russia \\ ${ }^{b}$ Institute of Mechanics and Applied Mathematics, Rostov State University, \\ pr. Stachki 200/1, Rostoy-on-Don, 344090 Russia \\ ${ }^{c}$ Institute for Ion Beam Physics and Materials Research Forschungszentrum Rossendorf, \\ P.O. Box 510119, D-01314, Dresden, Germany \\ ${ }^{d}$ Research Institute of Physics, Rostov State University, pr. Stachki 194, Rostov-on-Don, 344090 Russia \\ *e-mail:misochko@issp.ac.ru
}

\begin{abstract}
The relaxation dynamics of charge carriers in an epitaxial $\mathrm{La}_{0.7} \mathrm{Ca}_{0.3} \mathrm{MnO}_{3}$ film was studied by the light reflection method with a femtosecond time resolution in a wide temperature range. The relaxation of timeresolved photoinduced reflections was shown to be two-component in the whole temperature range including the paramagnetic dielectric-ferromagnetic metal transition at $T \approx 150-160 \mathrm{~K}$. The fast relaxation component had a maximum lifetime $(\tau \sim 500 \mathrm{ps})$ in the transition region, and its contribution to relaxation increased as temperature decreased. The lifetime of the slow component was minimum $(\tau \sim 15 \mathrm{fs})$ in the transition region. In addition, fast oscillations assigned to coherent phonons appeared in photoinduced responses at $T<200 \mathrm{~K}$. The dephasing time of these oscillations increased as temperature decreased, whereas their frequency changed insignificantly.
\end{abstract}

\section{INTRODUCTION}

The discovery of giant magnetoresistance in perovskite manganese oxides (such as $\mathrm{La}_{1-x} \mathrm{~A}_{x} \mathrm{MnO}_{3}$, where $\mathrm{A}=\mathrm{Sr}, \mathrm{Ca}$, or $\mathrm{Ba}$ ) has stimulated extensive studies of these systems. This interest in manganites is caused by at least two reasons. First, the giant magnetoresistance effect is potentially important for several technical applications, such as the design of magnetic recording heads and devices for storing information. Secondly, perovskite manganites are of interest for basic science. Unlike standard (elemental and oxide) ferromagnets, in which the electron spin is weakly coupled with the crystal lattice, manganites are characterized by mutually related spin, charge, orbital, and lattice degrees of freedom, which results in a fine balance of interactions responsible for their complex phase diagram [1]. This phase diagram includes metal-dielectric transitions, charge and orbital ordering, and electronic phase separation, which manifests itself by the formation of spin and charge stripes and other inhomogeneous states. The phase diagram of manganites is in many respects similar to the diagram of high- $T_{\mathrm{c}}$ superconductors, because materials of both classes are strongly correlated systems in which the potential energy of carriers is comparable with or larger than their kinetic energy. The con- ductivity (charge transport) of manganites can to a great extent be determined by the spin subsystem and caused by the double exchange mechanism, whereas the JahnTeller distortion of $\mathrm{Mn}^{3+} \mathrm{O}_{6}$ octahedra couples the spin and lattice degrees of freedom. The ferromagnetic transition in manganites is unusual and, as a rule, coincides with the metal-dielectric transition, which is responsible for the giant magnetoresistance effect. The results of experimental studies of these materials are summarized in review [1], and the most important theoretical models are described in reviews [2-5]. An analysis of these works shows that, currently, no consensus has been attained in understanding the physics of manganites. This makes further inquiries capable of providing new information a topical task.

Advances in laser technology, which allowed the laser pulse width to be reduced to several femtoseconds, opened up possibilities for studying the dynamics of excitation in solids in real time. At present, such studies are being actively performed for condensed media $[6,7]$. Understanding the mechanism of restoring equilibrium disturbed by a pumping pulse allows the special features of the dynamics of elementary excitations and their interaction to be revealed. Quite recently, the potential of optical time-resolved methods has been demonstrates in studies of such strongly cor- 
related systems as high- $T_{\mathrm{c}}$ superconductors, one- and two-dimensional systems with charge density waves, and one-dimensional perovskites [7-11]. This prompted us to undertake a study of one of the lanthanum manganites $\left(\mathrm{La}_{0.7} \mathrm{Ca}_{0.3} \mathrm{MnO}_{3}\right)$ by pumping-probing with femtosecond laser pulses to track the relaxation dynamics of quasi-particles in this material in a wide temperature range including the metal-insulator transition. Note that the creation of a nonequilibrium photoinduced hole population in high $-T_{\mathrm{c}}$ superconductors gave additional information about Hubbard constants in the copper and oxygen bands and allowed the charge gap width to be determined [12]. It follows from these results that a systematic study of manganites under the conditions when the electronic subsystem is nonequilibrium offers much promise.

\section{THE SAMPLES AND PROCEDURE FOR MEASUREMENTS}

The epitaxial $\mathrm{La}_{0.7} \mathrm{Ca}_{0.3} \mathrm{MnO}_{3}$ films were prepared by off-axis laser spraying on chemically purified $\mathrm{LaAlO}_{3}$ substrates $1.0 \times 1.0 \times 0.5 \mathrm{~cm}^{3}$ in size. The scheme for laser spraying was similar to that described in [13]. YAG : $\mathrm{Nd}^{3+}$ laser radiation $(\lambda=1.06 \mu \mathrm{m}, \tau=$ $15 \mathrm{~ns}$, and $f=12.5 \mathrm{~Hz}$ ) was focused into a $0.2 \times 0.2 \mathrm{~cm}^{2}$ spot on the surface of a rotating ceramic $\mathrm{La}_{0.7} \mathrm{Ca}_{0.3} \mathrm{MnO}_{3}$ target of the stoichiometric composition at an angle of $45^{\circ}$. The energy density on the surface of the target was about $2 \mathrm{~J} / \mathrm{cm}^{2}$. The oxygen pressure during deposition was $10-30 \mathrm{~Pa}$, and the distance between the target and the substrate was $3.5-4.5 \mathrm{~cm}$. The substrate temperature during film growth was varied in the temperature range $700-760^{\circ} \mathrm{C}$. The film thickness exceeded $1000 \AA$, and the lattice constant was $3860 \AA$. The X-ray patterns of the films only contained (001) $\mathrm{La}_{0.7} \mathrm{Ca}_{0.3} \mathrm{MnO}_{3}$ reflections and were free of traces of $h k l$-type orientations with $h, k \neq 0$ or impurity phases. The [100] $\mathrm{La}_{0.7} \mathrm{Ca}_{0.3} \mathrm{MnO}_{3} \|[100] \mathrm{LaAlO}_{3}$ orientation was observed in the substrate plane. The temperature dependence of the resistance of the samples was studied to find that, at $T=140-160 \mathrm{~K}$, the $\partial \rho / \partial T$ derivative changed sign from positive at low temperatures to negative at $T>150-160 \mathrm{~K}$, see inset to Fig. 1 . The electric conductivity, although small in magnitude, increased as the temperature rose. The conductivity changed by one to two orders of magnitude after the transition and decreased toward its low-temperature value as the temperature increased further. Measurements performed in an $H=0.9 \mathrm{~T}$ magnetic field showed that, at the highest film magnetoresistance, $\left(\rho_{H}-\rho_{0}\right) / \rho_{H} \approx 15 \%$.

Measurements in the time range were performed using a system for rapid scanning [9] and sapphire titanate laser pulses $(\lambda=780 \mathrm{~nm})$ width $70 \mathrm{fs}$ in width with a 78-MHz repetition frequency. The train of laser pulses was split into two beams. The probing beam was approximately 50 times weaker than the pumping beam (the probing beam power was $2 \mathrm{~mW}$ ). The sample was

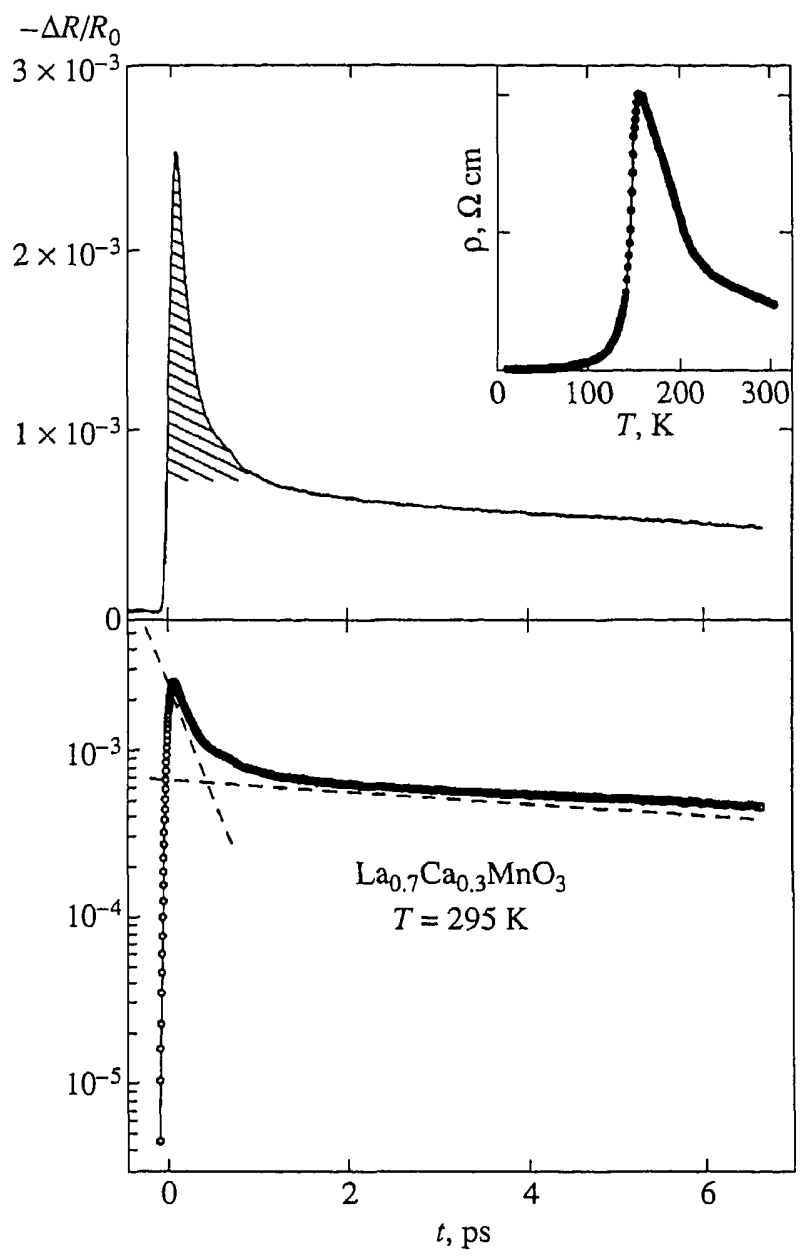

Fig. 1. Time-resolved differential reflection $\left|\Delta R / R_{0}\right|$ for a $\mathrm{La}_{0.7} \mathrm{Ca}_{0.3} \mathrm{MnO}_{3}$ film at room temperature. The fast relaxation component is hatched. Given in the inset is the temperature dependence of resistance. The dashed lines refer to the fast and slow relaxation channels.

excited by a pumping pulse and probed by the second (probing) pulse with a controlled time lag. The polarizations of the exciting and probing pulses were mutually orthogonal and lay in the film plane. The experimentally measured value was the difference reflection of the excited and unexcited samples $\Delta R(t)=R(t)-R_{0}$ as a function of delay time $t$ between the pumping and probing pulses. The zero point of the time axis was determined from the autocorrelation function. The sample was placed into an optical helium cryostat, which allowed us to perform measurements in the temperature range 4-330 $\mathrm{K}$.

\section{EXPERIMENTAL RESULTS AND DISCUSSION}

A typical optical response recorded by exciting and subsequently probing a $\mathrm{La}_{0.7} \mathrm{Ca}_{0.3} \mathrm{MnO}_{3}$ epitaxial film 


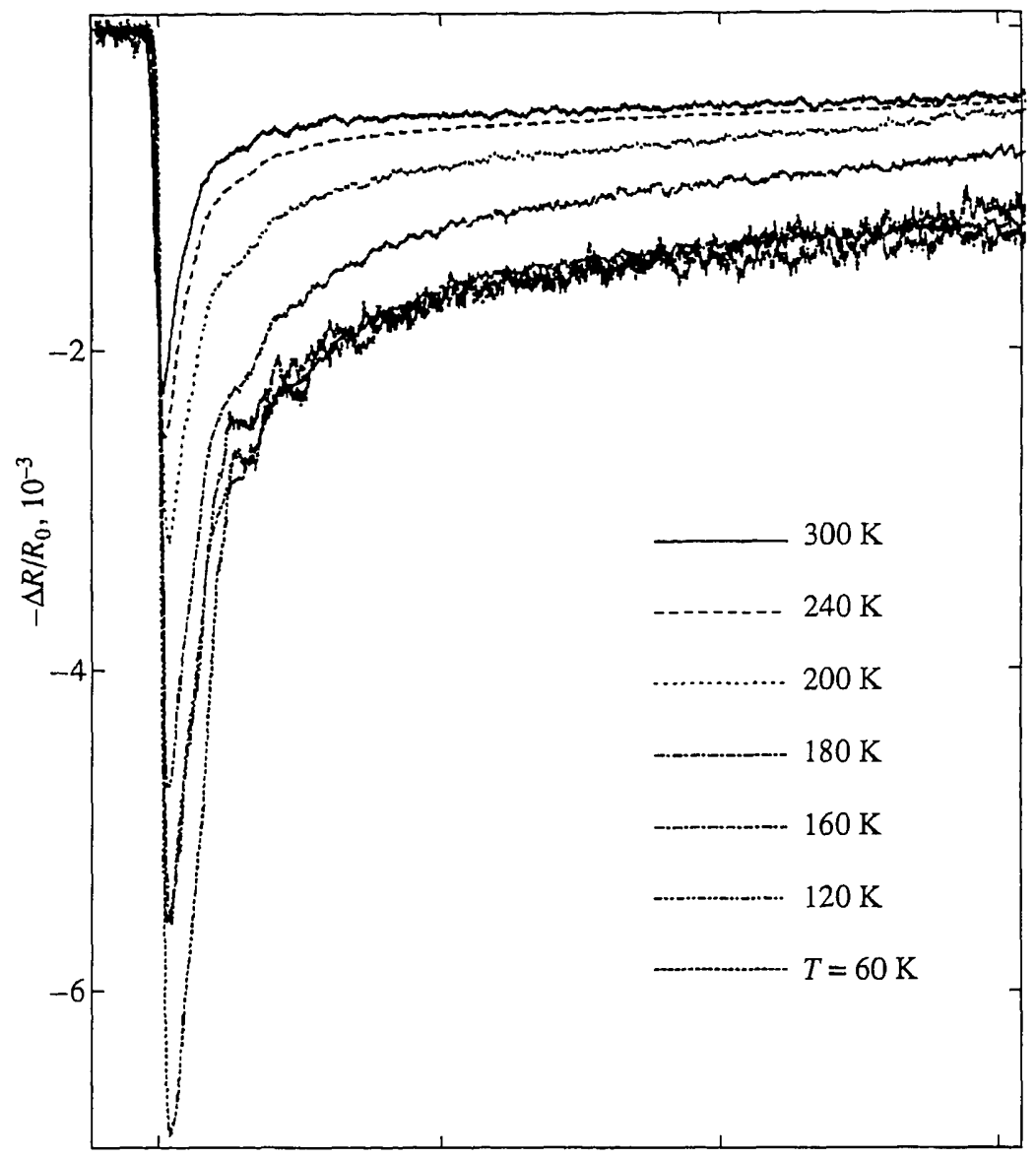

Fig. 2. Temperature dependences of time-resolved differential reflection $\Delta R / R_{0}$ at several specified temperatures.

by a femtosecond pulse is shown in Fig. 1. The film experiences a transition to the excited state in times on the order of 100-150 fs after the arrival of the pumping pulse. Simultaneously, its reflection decreases to its minimum value. This excited state on the system relaxes to the equilibrium state in times of the order of dozens of picoseconds. As the material under study has hole conductivity, its reflection decreases after the action of a pumping pulse. The relaxation of the photoinduced differential reflection is nonexponential in character and can be approximated by the sum of two (slow and fast) exponential functions,

$$
-\Delta R / R_{0}=A(T) \exp \left(-t / \tau_{A}\right)+B(T) \exp \left(-t / \tau_{B}\right) .
$$

This two-component relaxation dynamics is clearly seen in Fig. 1, in which the differential reflection is plotted on the linear and logarithmic scales. The fast component relaxes in times on the order of hundreds of femtoseconds, whereas the lifetime of the slow component amounts to dozens picoseconds.
The relaxation dynamics is shown in Fig. 2 for several temperatures in both the dielectric (paramagnetic) and metallic (ferromagnetic) states. This figure illustrates the temperature dependence of the time-resolved photoinduced response. The two-component relaxation dynamics is observed at all temperatures of our experiments, and the differential photoinduced response $\left|\Delta R / R_{0}\right|$ monotonically increases in magnitude as temperature decreases. In addition, the ratio between the fast and slow relaxation dynamics components changes. The lifetime of the fast component and its contribution to relaxation increase as the temperature approaches that of the largest resistance. Simultaneously, the lifetime of the slow component decreases and becomes minimum in the region of the paramagnetic dielectric-ferromagnetic metal transition temperature. In the ferromagnetic metallic phase, cooling the sample decreases the lifetime of the fast component and slightly increases the lifetime of the slow component. The results that describe temperature-induced changes in the dynamics of relaxation are summarized in Fig. 3. Note that $\Delta R$ reflection changes induced by the pump- 


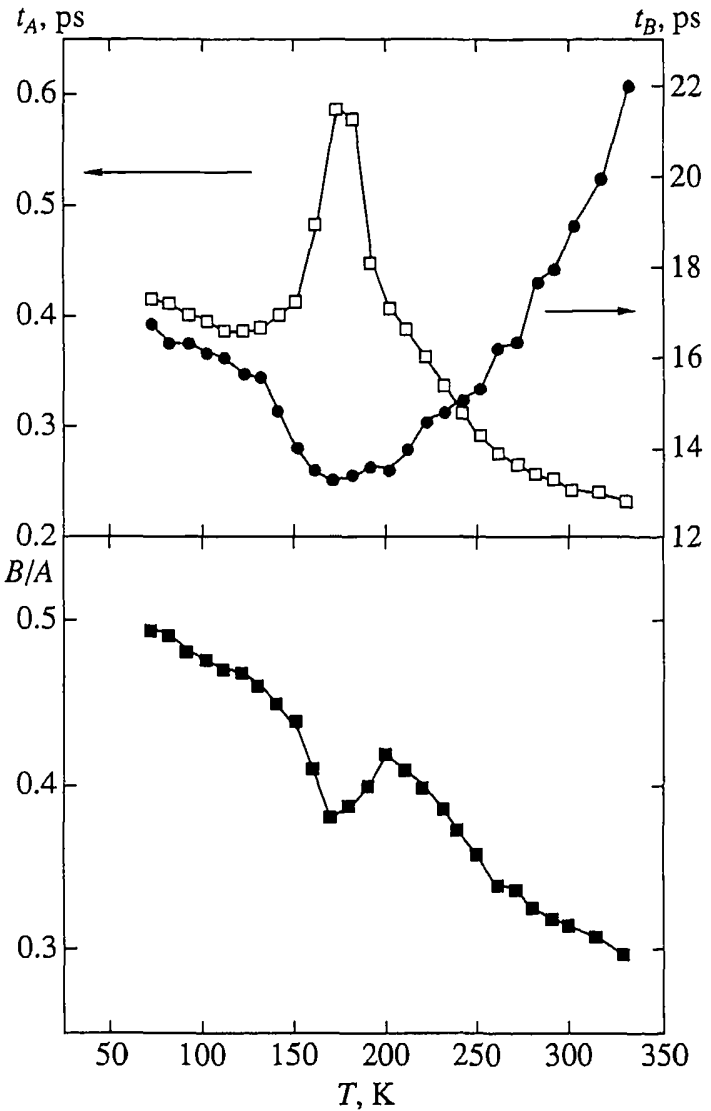

Fig. 3. Temperature dependences of the dynamic characteristics of excitation relaxation. Fast $\tau_{A}$ and slow $\tau_{B}$ relaxation components are given at the top, and the ratio between the fast and slow component contributions, at the bottom.

ing pulse are virtually independent of temperature. Changes in the $\Delta R / R_{0}$ differential reflection caused by sample cooling are largely determined by changes in the unexcited sample reflection $R_{0}$.

Somewhat above the temperature at which resistance is maximum, fast relaxation curve oscillations appear. These oscillations are shown in Fig. 4, where the nonoscillating relaxation component is subtracted. The oscillations can be attributed to coherent phonons excited by ultranarrow laser pulses [7]. The phonons recorded using subpicosecond pulses are called coherent because they have a well-defined phase [7], unlike thermal phonons, which are studied by Raman and infrared reflectance spectroscopy. Such phonons can appear because the exciting pulse width $\Delta t$ is smaller than the inverse phonon mode frequency $\Omega^{-1}, \Delta t<\Omega^{-1}$. Coherent phonons are recorded for $\mathrm{La}_{0.7} \mathrm{Ca}_{0.3} \mathrm{MnO}_{3}$ films at all temperatures below $T=200 \mathrm{~K}$ and are absent at the higher temperatures. The phonon modes were identified by numerically Fourier-transforming the time range data into the frequency range; the result is shown in Fig. 5. The period of the oscillations insig-

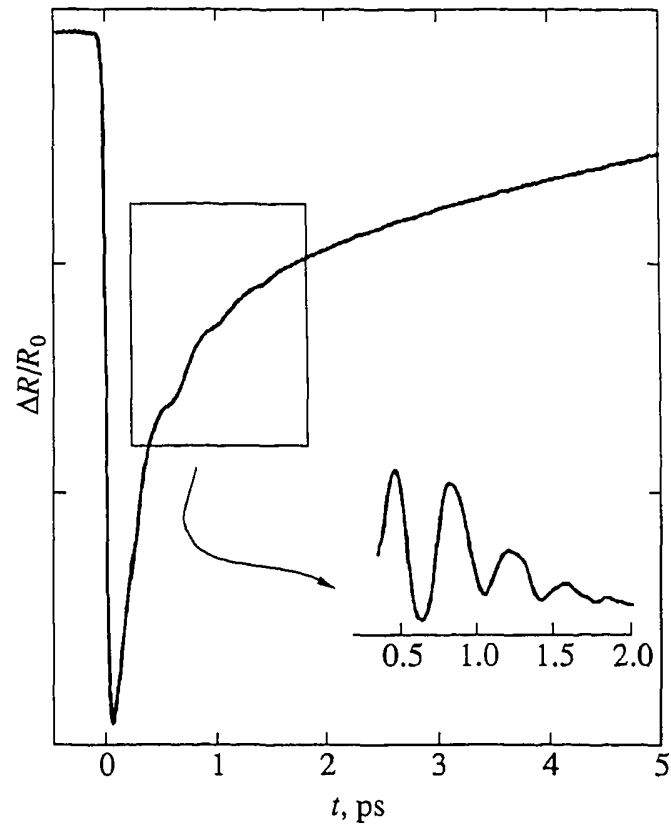

Fig. 4. Time resolved differential reflection $\Delta R / R_{0}$ of a $\mathrm{La}_{0.7} \mathrm{Ca}_{0.3} \mathrm{MnO}_{3}$ film at $T=150 \mathrm{~K}$. Shown in the inset are the oscillations assigned to coherent phonons.

nificantly decreases and their lifetime considerably increases as the temperature lowers. For instance, at $T=190 \mathrm{~K}$, the oscillations are damped during one cycle, whereas the frequency of the oscillations at $T=$ $150 \mathrm{~K}$ is much higher than the inverse damping time. This follows from a comparison of both time-resolved responses and periodograms of the oscillating response component. One of such periodograms is shown in Fig. 5.

It is expedient to precede a possible interpretation of the results with a presentation of the generally accepted views on the system under study. The $\mathrm{La}_{0.7} \mathrm{Ca}_{0.3} \mathrm{MnO}_{3}$ compound can be treated as a solid solution formed by $\mathrm{LaMnO}_{3}$ and $\mathrm{CaMnO}_{3}$. Doping $\mathrm{LaMnO}_{3}$ with divalent $\mathrm{Ca}$ yields a mixed-valence compound, which contains $\mathrm{Mn}^{3+}$ and $\mathrm{Mn}^{4+}$ ions. In the double exchange model, the width of the hole band, which depends on the level of doping and temperature, controls the metal-dielectric transition. Exchange coupling alone is, however, insufficient for obtaining the real characteristics of manganites. As distortions caused by the cooperative JahnTeller effect already exist in the initial $\mathrm{LnMnO}_{3}$ compound and lower its symmetry from cubic, it has been suggested that taking into account lattice excitations and strong electron-phonon coupling may give a correct description of the system. The Jahn-Teller effect lowers crystal symmetry and removes degeneracy of the $e_{g} d \mathrm{Mn}$ orbitals. Depending on the theoretical model, it is assumed that doping results in the appearance of a hole either on the manganese $d$ orbital [4] or 


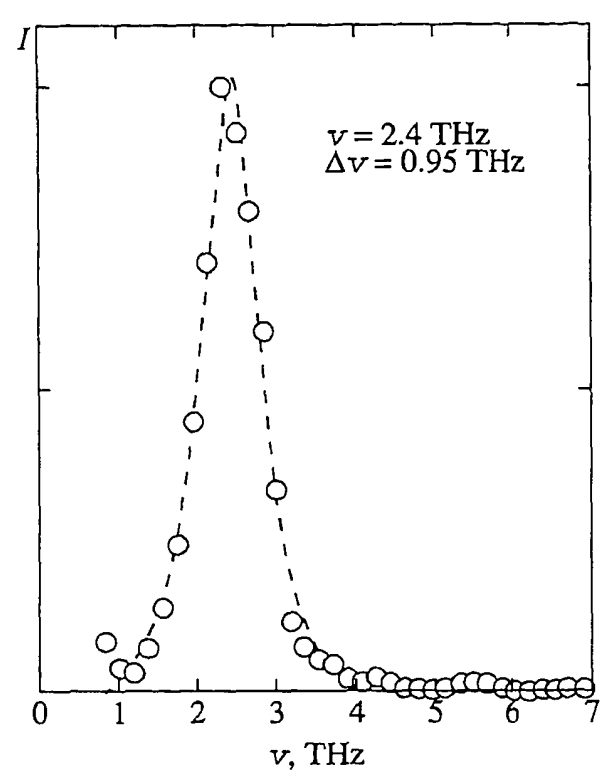

Fig. 5. Fourier transform of the time response of the oscillating $\Delta R / R_{0}$ signal part at $T=150 \mathrm{~K}$. The dashed line was obtained by fitting the Lorentzian function with the parameters shown in the figure to the experimental curve.

on the oxygen $p$ orbital [3]. Irrespective of the model, it is, however, believed that the conductivity of this system is polaron in character (that is, the charge carrier is "dressed" in a phonon or magnetic coat).

We begin the discussion with the temperature dependence of the differential reflection, which increases as the temperature lowers. An increase in $\left|\Delta R / R_{0}\right|$ is, as mentioned above, caused by a decrease in $R_{0}$, that is, in reflection at negative delay times $t$. A decrease in $R_{0}$ at low temperatures is determined by changes in the optical spectra. It is known that, in the dielectric phase, the optical conductivity spectra have a peak at $\approx 1.0 \mathrm{eV}$, which increases in intensity and shifts to the lower frequencies as the temperature decreases. This peak is also recorded in the ferromagnetic phase and transforms into a Drude response at helium temperatures $[14,15]$. The temperature dependence of the optical conductivity was described using the double exchange model including the dynamic Jahn-Teller effect; it was attributed to changes in the Jahn-Teller polaron, which had a small radius at high temperatures and a large radius at low temperatures [14]. The appearance of the quasi-Drude response in the temperature range in which the resistance is fairly large was, however, explained in [15] by phase separation (according to this model, charge carriers experienced segregation in high-conductivity regions, which had the form of ferromagnetic drops in a dielectric matrix). Inelastic neutron scattering measurements gave experimental evidence for phase stratification in manganites; in these experiments, two spin excitation branches with qua- dratic and linear dispersion laws were recorded [1618]. The first branch was associated with magnons in the ferromagnetic phase, and the second branch was assigned to magnetic excitations of the antiferromagnetic phase.

If, following [15], we relate the fast relaxation component to the relaxation of carriers in the ferromagnetic phase (which are characterized by high mobility and low concentration) and the slow component to carriers in the antiferromagnetic phase (low mobility and high concentration), then the fraction of the ferromagnetic phase should decrease as the temperature lowers because the $B / A$ ratio increases. Such a behavior is physically meaningless, because the fraction of the ferromagnetic phase at $T<T_{\mathrm{c}}$ cannot be smaller than at $T>T_{\mathrm{c}}$.

One can attempt to relate the fast component of the decay of the excited state to the relaxation of photoexcited band carriers, whereas the slow component may be ascribed to the relaxation of the polaron coat, which is responsible for the large mass of polarons and consists of localized spins and/or phonons. With such an assignment of the components, a change in the temperature dependences of the components in the region of the transition temperature is, most likely, evidence of the crossover of the nature of the polarons. This can be the transition from large-sized to small-sized polarons or a bipolaron-polaron-type transition [3].

We emphasize that we do not assert that the twocomponent response to the action of ultranarrow laser pulses cannot be explained within the framework of the phase separation model. The optical method that we use is, however, an integral method, and the question of the characteristic size of nonuniformities and the topology of the nonuniform state therefore remains open. We can only note that the films were macroscopically homogeneous and their relaxation dynamics was not influenced by observation point displacements (the size of the probed region was $300 \mu \mathrm{m}$ ).

Note that several experimental and theoretical works give evidence of the thermally activated character of conductivity for optimally doped manganites in the paramagnetic phase $[19,20]$. A detailed timeresolved study of manganites at temperatures $T \geq T_{c}$ can in principle be used to estimate the activation energy.

The fast photoinduced response oscillations occur at a frequency of $2.4 \mathrm{THz}\left(=80 \mathrm{~cm}^{-1}\right)$. The low oscillation frequency favors the assignment of this phonon to either La-ion stretching motions or oxygen octahedron rotations ( $\mathrm{Mn}$ ions make no contribution to even phonons, and the stretching and bending oxygen motions have much higher frequencies). Unfortunately, we cannot directly compare our results with Raman spectra because data on the Raman frequencies below $100 \mathrm{~cm}^{-1}$ are lacking [21]. In principle, an arbitrary Raman-active (even) phonon can be excited by femtosecond pulses provided the excitation mechanism is stimulated Raman scattering. If coherent phonons are 
generated by the shift mechanism, totally symmetrical modes can only be observed [16]. As the coherent phonon with a $2.4 \mathrm{THz}$ frequency exists both above and below the transition temperature, it should be suggested that the transition occurs without a change in lattice symmetry. The fast photoinduced response oscillations are absent at temperatures $T>200 \mathrm{~K}$ because the decay of this mode is a strong function of temperature and the phonon is predamped in this temperature range. At the same time, there is no indication of transition-induced phonon frequency softening. Some increase in the frequency at low temperatures is most likely caused by lattice anharmonicity.

\section{CONCLUSIONS}

The temperature dependence of relaxation dynamics is evidence that two contributions with substantially different lifetimes exist in $\mathrm{La}_{0.7} \mathrm{Ca}_{0.3} \mathrm{MnO}_{3}$ lanthanum manganites. The fast relaxation component with lifetimes of the order of hundreds of femtoseconds reaches a maximum in the region of the dielectric-metal transition, and its contribution increases as temperature decreases. The slow component with lifetimes of the order of dozens of picoseconds also has an extremum (minimum) in the transition region. The two-component character of relaxation, which includes contributions whose ratio changes in the transition, is evidence of a change in the character of the polaron responsible for conductivity in this material.

The fast photoinduced response oscillations assigned to coherent phonons exist both above and below the transition temperature. It follows that the structure of the lattice remains unchanged in the transition. However, note that, although the frequency of these oscillations changes insignificantly as temperature decreases, their damping (dephasing) considerably weakens.

\section{ACKNOWLEDGMENTS}

This work was financially supported by the Russian Foundation for Basic research (project no. 01-02-1640), a NATO grant for joint scientific research (PST.GLG.978935), and the Humboldt Foundation (Germany).

\section{REFERENCES}

1. J. M. D. Coey, M. Virtel, and S. von Molnar, Adv. Phys. 48, 167 (1999).

2. É. L. Nagaev, Usp. Fiz. Nauk 166, 577 (1996) [Phys. Usp. 39, 539 (1996)].

3. A. S. Alexandrov and A. M. Bratkovsky, J. Phys.: Condens. Matter 11, 1989 (1999).

4. Yu. A. Izyumov and Yu. N. Skryabin, Usp. Fiz. Nauk 171, 121 (2001) [Phys. Usp. 44, 109 (2001)].

5. M. Yu. Kagan and K. I. Kugel', Usp. Fiz. Nauk 171, 833 (2001).

6. J. Shah, Ultrafast Spectroscopy of Semiconductors and Semiconducting Nanostructures (Springer, Telos, 1998).

7. O. V. Misochko, Zh. Éksp. Teor. Fiz. 119, 285 (2001) [JETP 92, $246(2001)]$.

8. J. Demsar, L. Forro, H. Berger, and D. Mihailovic, Phys. Rev. B 66, 041101 (2002).

9. T. Ogasawara, M. Ashida, N. Motoyama, et al., Phys. Rev. B 63, 113105 (2001).

10. J. Demsar, K. Biljakovic, and D. Mihailovic, Phys. Rev. Lett. 83, 800 (1999).

11. O. V. Misochko, N. Georgiev, T. Dekorsy, and M. Helm, Pis'ma Zh. Éksp. Teor. Fiz. 75, 768 (2002) [JETP Lett. 75, $642(2002)]$.

12. V. I. Kudinov, A. I. Kirilyuk, N. M. Kreines, et al., Phys. Lett. A 151, 358 (1990).

13. E. Kaidashev, V. Dneprovski, D. Breus, and R. N. Sheftal, J. Supercond. 13, 407 (2000).

14. M. Quijada, J. Cerne, J. R. Simpson, et al., Phys. Rev. B 58, 16093 (1998).

15. N. N. Loshkareva, Yu. P. Sukhorukov, V. E. Arkhipov, et al., Fiz. Tverd. Tela (St. Petersburg) 41, 475 (1999) [Phys. Solid State 41, 426 (1999)].

16. E. O. Wollan and W. C. Koehler, Phys. Rev. 100, 545 (1955).

17. M. Hennion, F. Moussa, G. Biotteau, et al., Phys. Rev. Lett. 81, 1957 (1998).

18. Y. Moritomo, A. Machida, S. Mori, et al., Phys. Rev. B 60, 9220 (1999).

19. A. L. Rakhmanov, K. I. Kugel, Ya. M. Blanter, and M. Yu. Kagan, Phys. Rev. B 63, 174424 (2001).

20. J. H. Zhao, H. P. Kunkel, X. Z. Zhou, and Gwyn Williams, Phys. Rev. B 66, 184428 (2002).

21. L. Martin-Carron, A. De Andres, J. Martinez-Lope, et al., Phys. Rev. B 66, 174303 (2002).

Translated by V. Sipachev 